\title{
CONF-970678--1
}

SANO97.0730C

\section{Fractal Functions and Multiwavelets}

\author{
Peter R. Massopust ${ }^{1}$ \\ Applied and Numerical Mathematics Department \\ Sandia National Laboratories \\ Albuquerque, NM 87185-1110 \\ U.S.A.
}

\begin{abstract}
This paper reviews how elements from the theory of fractal functions are employed to construct scaling vectors and multiwavelets. Emphasis is placed on the one-dimensional case, however extensions to $\mathbb{R}^{m}$ are indicated.
\end{abstract}

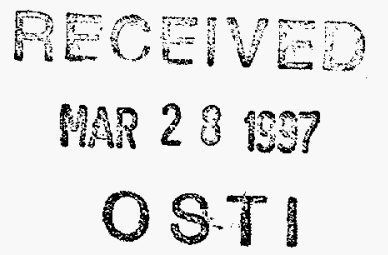

\section{Construction in $\mathbb{R}$}

Let $\Omega:=[0,1)$ and let $\mathbb{Z}_{N}:=\{0,1, \ldots, N-1\}$ with $1<N \in \mathbb{N}$. For $\ell \in \mathbb{Z}_{N}$, let $\lambda_{\ell} \in P_{d}(\Omega)$, the space of real polynomials of degree at most $d$ on $\Omega$, and $s_{\ell} \in(-1,1)$. Set $\lambda:=\left(\lambda_{\ell}\right)_{\ell \in \mathbb{Z}_{N}} \in \Theta_{d}(\Omega):=\Pi_{\ell \in \mathbb{Z}_{N}} P_{d}(\Omega)$ and $s:=\left(s_{\ell}\right)_{\ell \in \mathbb{Z}_{N}}$. Then the operator $B=B(\lambda, s): L^{\infty}(\Omega) \rightarrow L^{\infty}(\Omega)$ defined by

$$
B f:=\sum_{\ell \in \mathbb{Z}_{N}}\left(\lambda_{\ell}(N \cdot-\ell)+s_{\ell} f(N \cdot-\ell)\right) \chi_{[\ell / N,(\ell+1) / n)} .
$$

is contractive on $\left(L^{\infty}(\Omega),\|\cdot\|_{L^{\infty}}\right)$, and its unique fixed point $f^{*}=f_{\lambda, s}^{*}$ satisfies

$$
f^{*}=\Lambda+\sum_{\ell \in \mathbb{Z}_{N}} s_{\ell} f^{*}(N \cdot-\ell)
$$

Here $\Lambda: \mathbb{R} \rightarrow \mathbb{R}$ is defined by $\left.\Lambda\right|_{[\ell / N,(\ell+1) / n)}:=\lambda_{\ell}(N \cdot-\ell), \ell \in \mathbb{Z}_{N}$, and $\Lambda: \equiv 0$ on $\mathbb{R} \backslash \Omega$. (Note that $f^{*}$ has been extended to all of $\mathbb{R}$ be setting $f^{*} \equiv 0$ outside $\Omega$.) If, in addition, the interior boundary conditions $\lambda_{\ell+1}(0)+s_{\ell+1} f(0)=$ $\left.\lambda_{\ell}(1-)+s_{\ell} f(1-)\right), \ell \in \mathbb{Z}_{N}$, hold, then $B$ is an operator on $L^{\infty}(\Omega) \cap C(\bar{\Omega})$.

The function $f^{*}$ is called a fractal function. The graph of $f^{*}$ is made up of a finite number of copies of itself and is, in general, a fractal set (cf. $[1,6]$ ). This above-defined class of fractal functions is denoted by $\mathcal{P}_{d}(\Omega)$.

Equations of type (2) are referred to as inhomogeneous refinement equations since the function $f^{*}$ is expressed in terms of a refined version $f^{*}(N \cdot-\ell)$ of itself.

The next theorem gives the relationship between $\lambda$ and the fractal functions $f^{*} \in \mathcal{P}_{d}(\Omega)$

\footnotetext{
${ }^{1}$ Research partially supported by NSF grant DMS-9503282. This work was also supported by Sandia National Laboratories. Sandia is a multiprogram laboratory operated by Sandia Cooperation, a Lockheed Martin Company, for the United States Department of Energy (DOE) under Contract DE-AC04-94AL85000.
} 


\section{DISCLAIMER}

This report was prepared as an account of work sponsored by an agency of the United States Government. Neither the United States Government nor any agency thereof, nor any of their employees, make any warranty, express or implied, or assumes any legal liability or responsibility for the accuracy, completeness, or usefulness of any information, apparatus, product, or process disclosed, or represents that its use would not infringe privately owned rights. Reference herein to any specific commercial product, process, or service by trade name, trademark, manufacturer, or otherwise does not necessarily constitute or imply its endorsement, recommendation, or favoring by the United States Government or any agency thereof. The views and opinions of authors expressed herein do not necessarily state or reflect those of the United States Government or any agency thereof. 


\section{DISCLAMMER}

Portions of this document may be illegible in electronic image products. Images are produced from the best available original document. 
Theorem 1.1 The mapping $\vartheta: \Theta_{d}(\Omega) \rightarrow L^{\infty}(\Omega), \lambda \mapsto f_{\lambda}^{*}$, is a linear isomorphism.

The interested reader may consult [4] or [6] for a proof.

Indeed, more can be shown if $d=1$ and $f^{*} \in L^{\infty}(\Omega) \cap \widetilde{C}_{\mathbb{R}}(\Omega)$, where $\widetilde{C}_{\mathbb{R}}(\Omega):=\left\{\left.f\right|_{\Omega}: f \in C(\bar{\Omega})\right.$. Since in this case each vector $\boldsymbol{y}:=\left(y_{0}, y_{1}, \ldots, y_{N}\right)^{T}$ $\in \mathbb{R}^{N+1}$ uniquely determines a $\lambda \in \Pi_{\ell \in \mathbb{Z}_{N}} P_{1}(\Omega) \subseteq \Theta_{1}(\Omega)$, and vice versa, the mapping $\mathbb{R}^{N+1} \ni \boldsymbol{y} \mapsto \lambda(\boldsymbol{y}) \in \Theta_{1}(\Omega)$ is a linear isomorphism. Hence, the following result holds.

Theorem 1.2 The mapping $\eta: \mathbb{R}^{N+1} \rightarrow L^{\infty}(\Omega) \cap \widetilde{C}_{\mathbb{R}}(\Omega), y \mapsto f_{\lambda(y)}^{*}$, is a linear isomorphism.

This result provides a geometric interpretation of fractal functions: Every fractal function $f^{*} \in \mathcal{P}_{1}(\Omega)$ is uniquely determined by an interpolation vector $y \in$ $\mathbb{R}^{N+1}$, i.e., by its values at a given set of knot points $\left\{x_{i}\right\}_{i=0}^{N}$. Hence the linear space $\mathcal{P}_{1}(\Omega)$ of fractal functions on $\Omega$ is $N+1$ dimensional and a canonical basis is given by the fractal functions $f_{i}^{*}:=\eta\left(\boldsymbol{y}_{i}\right)$, where $\boldsymbol{y}_{i}(j)=\delta_{i j}, i, j=0,1, \ldots, N$.

The fractal functions in $\mathcal{P}_{d}(\Omega)$ may be used to construct finitely generated shift-invariant subspaces of $L^{2}(\Omega)$ (cf., for instance, $[4,6]$ ). Recall that one of the main features of a fractal function $f^{*} \in \mathcal{P}_{d}(\Omega)$ is that $f^{*}(\cdot / 2)$ restricted to $\Omega$ is again a fractal function. More precisely, if $f^{*}$ is generated by $\lambda \in \Theta_{d}(\Omega)$, then $f^{*}(\cdot / 2)$ restricted to $[\ell / 2,(\ell+1) / 2)$ is generated by $\lambda(\ell):=\left(\lambda_{\ell, \ell^{\prime}}\right)_{\ell^{\prime} \in \mathbb{Z}_{N}} \in$ $\Theta_{d}(\Omega)$, where $\lambda_{\ell, \ell^{\prime}}:=\lambda_{\ell}\left(\left[\cdot-\ell^{\prime}\right] / N\right)+s_{\ell^{\prime}} \lambda_{\ell^{\prime}}-s_{\ell} \lambda_{\ell}, \ell, \ell^{\prime} \in \mathbb{Z}_{N}$. This observation allows the construction of piecewise fractal functions. For a given $i \in I \subseteq \mathbb{Z}$, construct a fractal function $f_{i} \in \mathcal{P}_{d}([i, i+1))$. Denote by $\lambda(i):=\left(\lambda_{i, \ell}\right)_{\ell \in \mathbb{Z}_{N}}$ the corresponding element in $\Theta_{d}([i, i+1))$. Let $f:=\left\{f_{i}: i \in I\right\}$. Every sequence $\lambda:=\{\lambda(i): i \in I\} \in \prod_{i \in \mathbb{Z}} \Theta_{d}([i, i+1))$ defines a $f \in \prod_{i \in I} L^{\infty}([i, i+1))$. An element $f \in \prod_{i \in I} L^{\infty}([i, i+1))$ may then be identified with a function $f \in L^{\infty}(\mathbb{R})$ via the linear isomorphism

$$
f \stackrel{\tau}{\longmapsto} \sum_{i \in I} f_{i} \chi_{[i, i+1)}
$$

The representation of $\boldsymbol{f}$ under $\tau$ is called a piecewise fractal function.

Now let $\left\{\mathcal{O}_{i}\right\}_{i \in I}$ be a countable collection of mutually disjoint open sets of positive measure such that $\bigcup_{i \in I} \overline{\mathcal{O}}_{i}=\mathbb{R}$. Define $C_{\mathcal{O}}(\mathbb{R}):=\{f \in C(\mathbb{R}): f$ is bounded on $\left.\overline{\mathcal{O}}_{i}, i \in I\right\}$. Then $C_{\mathcal{O}}$ becomes a Banach space when endowed with the norm $\|f\|_{C_{0}}:=\sup _{i \in I}\left\{|f(x)|: x \in \mathcal{O}_{i}\right\}$. Using methods from $[4,6]$ and choosing $\mathcal{O}_{i}:=(i, i+1), I:=\mathbb{Z}$, one can show that the sequence of shiftinvariant spaces $\left\{V_{k}\right\}_{\mathbb{Z}}$ defined by

$V_{0}:=\left\{g \in C_{\mathcal{O}}(\mathbb{R}):\left.\exists f \in \mathcal{P}_{n+1}([i, i+1)) \cap C(\Omega){ } g\right|_{(i, i+1)}=\left.f\right|_{(i, i+1)}\right\} \cap L^{2}(\mathbb{R})$,

and $f \in V_{k} \Longleftrightarrow f\left(2^{k} \cdot\right) \in V_{0}$, satisfies $V_{k-1} \subset V_{k}, k \in \mathbb{Z}, \bigcap_{k \in \mathbb{Z}} V_{k}=\{0\}$, and $\bigcup_{k \in \mathbb{Z}} V_{k}=L^{2}(\mathbb{R})$. The following theorem summarizes results obtained in $[4,6]$. 
Theorem 1.3 1. There exist two piecewise fractal functions $\phi^{0}$ and $\phi^{1}$ generated by affine maps $\left(\lambda_{\ell}\right)_{\ell=0}^{1}$ and the set of parameters $s_{0}=s_{1}=-1 / 5$ such that $V_{0}=\overline{\operatorname{span}\left\{\phi^{\ell}(\cdot-n): \ell=0,1 ; n \in \mathbb{Z}\right\}}{ }^{L^{2}(\mathbb{R})}$.

2. The scaling vector $\phi:=\left(\phi^{0} \phi^{1}\right)^{T}$ is orthogonal to its integer translates: $\int_{\mathbb{R}} \phi(x) \phi^{T}(x-n) d x=\delta_{n} I$. (Here I denotes the identity matrix.)

3. The $L^{2}$-orthogonal complement, $W_{k+1}$, of $V_{k}$ in $V_{k+1}$ is generated by a multiwavelet $\psi:=\left(\psi^{0} \psi^{1}\right)^{T}$ where each component consists of four piecewise fractal functions generated by affine maps and parameters $s_{0}=s_{1}=$ $-1 / 5$.

4. $\forall k \in \mathbb{Z} \forall n \in \mathbb{Z} \int_{\mathbb{R}} \psi\left(2^{k} x\right) \psi^{T}\left(2^{k} x-n\right) d x=\delta_{n} I$.

5. $\operatorname{supp} \phi^{0}=[0,1], \operatorname{supp} \phi^{1}=\operatorname{supp} \psi^{0}=\operatorname{supp} \psi^{1}=[0,2]$. Moreover, $\psi^{0}$ is symmetric and $\psi^{1}$ antisymmetric about $x=1$.

6. The scaling vector $\phi$ has approximation order two and $\int_{\mathbb{R}} x^{\ell} \psi(x) d x=0$, $\ell=0,1$.

7. $\phi, \psi \in \times_{\ell=0}^{1} \operatorname{Lip}^{1}(\mathbb{R})$. In particular, $\phi, \psi \in \times \times_{\ell=0}^{1} H^{1}(\mathbb{R})$.

8. The scaling vector $\phi$ and the multiwavelet $\psi$ are interpolatory, in the sense that given a set of interpolation points $\left(i / 2^{k}, F_{i}\right), i=0,1, \ldots, 2^{k}$, there exist real vectors $c_{k j}$ such that $F_{i}=\sum_{j} c_{k j}^{T} \phi_{k j}\left(i / 2^{k}\right)$. (Here $\phi_{k j}:=$ $\phi\left(2^{k} \cdot-j\right)$.)

The scaling vector $\phi$ and associated multiwavelet $\psi$ may be employed to define a multiresolution analysis on $L^{2}(\Omega)$. To this end, let $\mathcal{B}_{k}^{\phi}(\Omega):=\left\{\left.\phi_{k j}^{\ell}\right|_{\Omega}: \ell=\right.$ 0,$\left.1 ;-\ell \leq j \leq 2^{k}-1 ; k \in \mathbb{Z}_{0}^{+}\right\}$and $\mathcal{B}_{k}^{\psi}(\Omega):=\left\{\left.\psi_{k j}^{\ell}\right|_{\Omega}: \ell=0,1 ; \ell-1 \leq j \leq\right.$ $\left.2^{k-1}-\ell+1 ; k \in \mathbb{Z}_{0}^{+}\right\}$.

Theorem $1.4 \mathcal{B}_{k}^{\phi}(\Omega)$ is an orthonormal basis for $V_{k}(\Omega):=V_{k} \cap L^{2}(\Omega)$ and $\mathcal{B}_{k}^{\psi}(\Omega)$ an orthonormal basis for $W_{k+1}(\Omega):=W_{k+1} \cap L^{2}(\Omega)$. Moreover, $L^{2}(\Omega)=$ $V_{0}(\Omega) \bigoplus_{k \in \mathbb{Z}_{0}^{+}} W_{k+1}(\Omega)$.

The proof may be found in [4] or [6].

\section{Extension to $\mathbb{R}^{m}$}

The above procedure to obtain multiwavelets may be extended to $\mathbb{R}^{m}$ by employing crystallographic Coxeter groups. The main idea is as follows. The domain $\Omega$ is now a convex polytope having $0 \in \mathbb{R}^{m}$ as a vertex and tesselating $\mathbb{R}^{m}$ by reflections in hyperplanes. It is well-known that such polytopes are 
in one-to-one correspondence with crystallographic Coxeter groups $\mathcal{W}$. For a given $N \in \mathbb{N}$, consider the convex polytope $\Sigma:=\left\{N^{m} \omega: \omega \in \Omega\right\}$. Then $\Sigma$ is tesselated by $N^{m}$ subpolytopes $\Sigma_{\ell}, \ell=0,1, \ldots, N^{m}-1$, one of which, say, $\Sigma_{0}$ is $\Omega$. The next theorem is proven in [5].

Theorem 2.1 Let $s=\left(s_{\ell}\right)_{\ell=0}^{N^{m}-1}$ be a given set of free parameters, let $\lambda=$ $\left(\lambda_{\ell}\right)_{\ell=0}^{N^{m}}-1 \in \times_{\ell=0}^{N^{m}-1} C(\Sigma, \mathbb{R})$ and let $f \in L^{\infty}(\Sigma, \mathbb{R})$. If $\max _{0 \leq \ell \leq N^{m}-1}\left|s_{\ell}\right|<1$, then the operator $B=B(\lambda, s): L^{\infty}(\Sigma, \mathbb{R}) \rightarrow L^{\infty}(\Sigma, \mathbb{R})$ defined by

$$
B f:=\sum_{\ell=0}^{N^{m}-1}\left[\lambda_{\ell}\left(N r_{\Sigma_{0} \Sigma_{\ell}}\right)+s_{\ell} f\left(N r_{\Sigma_{0} \Sigma_{\ell}}\right)\right] \chi_{\Sigma_{\ell}}
$$

where $r_{\Sigma_{0} \Sigma_{\ell}}$ is the unique element in $\mathcal{W}$ mapping $\Sigma_{\ell}$ onto $\Sigma_{0}$, is contractive on $\left(L^{\infty}(\Sigma, \mathbb{R}),\|\cdot\|_{L^{\infty}}\right)$.

The fixed points of $B$ are referred to as fractal surfaces. Analogously to the onedimensional case shift-invariant subspaces $V$ of $L^{2}\left(\mathbb{R}^{m}\right)$ can be constructed from propely pieced-together fractal surfaces. It should be noted that these function spaces are dilation invariant with respect to the scale $V \ni f \mapsto f(\cdot / N) \in V$ and $\mathcal{W}$-invariant in the sense that $V \ni f \mapsto f \circ r \in V, \forall r \in \mathcal{W}$. (Note that since the translations along the underlying lattice $\Gamma$ for $\mathcal{W}$ are contained in $\mathcal{W}$, any $\mathcal{W}$-invariant is also translation-invariant with respect to $\Gamma$.) The interested reader is referred to [5] for more details.

\section{References}

[1] Barnsley M. F. (1986): Fractal functions and interpolation. Constr. Approx. 2, 303-329

[2] Daubechies I. (1992): Ten Lectures on Wavelets. SIAM, Vol. 61, Philadelphia

[3] De Boor, C., DeVore, R., Ron, A. (1994): The structure of finitely generated shift-invariant spaces in $L_{2}(\mathbb{R})$. J. Funct. Anal. 119, 37-78

[4] Donavan, G., Geronimo, J. S., Hardin, D. P., Massopust, P.R. (1996): Construction of orthogonal wavelets using fractal interpolation functions. SIAM J. Math. Anal. 27(4), 1158-1192

[5] Geronimo, J. S., Hardin, D. P., Massopust, P.R. (1994): An application of Coxeter groups to the construction of wavelet bases in $\mathbb{R}^{n}$. (Lecture Notes in Pure and Applied Mathematics, Vol. 157), Marcel Dekker, New York

[6] Massopust, P. R. (1995): Fractal Functions, Fractal Surfaces, and Wavelets. Academic Press, Orlando 